\title{
Comparisons between 2D and 3D Uniform Array Antennas
}

\author{
Andy Vesa \\ POLITEHNICA University \\ Timişoara, Romania, \\ Communications Department, V \\ Parvan No 2, 300223, Timişoara \\ Email: andy.vesa@upt.ro
}

\author{
Florin Alexa \\ POLITEHNICA University \\ Timişoara, Romania, \\ Communications Department, V \\ Parvan No 2, 300223, Timişoara \\ Email: florin.alexa@upt.ro
}

\author{
Horia Baltă \\ POLITEHNICA University \\ Timişoara, Romania, \\ Communications Department, $\mathrm{V}$ \\ Parvan No 2, 300223, Timişoara \\ Email: horia.balta@upt.ro
}

\begin{abstract}
For any wireless communications antenna system becomes indispensable. In this paper we analyzed linear array, planar array and three - dimensional (3D) array antennas. The array systems are simulated in Matlab based on uniform linear array antennas. Comparisons between planar array antenna and 3D array antenna are provided take into account different phases of currents injected in antenna elements. Also we propose to use the array antenna in WSN due to the advantages in signal to noise ratio and power consumption.
\end{abstract}

\section{INTRODUCTION}

A radio antenna, transmitting or receiving, is an independent and yet integral component of any wireless communication system. An antenna acts as a transducer that converts the current or voltage generated by the feedingbased circuit, such as a transmission line, a waveguide or coaxial cable, into energy field propagating through space and vice versa. Each radio signal can be represented as an electromagnetic wave that propagates along a given direction. The wave field strength, its polarization and the direction of propagation determine the main characteristics of an antenna operation [1].

Antennas can be divided in different categories, such as wire antennas, aperture antennas, reflector antennas, frequency independent antennas, horn antennas, printed and conformal antennas, and so forth. When applications require radiation characteristics that cannot be met by a single radiating antenna, multiple elements are employed forming "array antennas". Arrays can produce the desired radiation characteristics by appropriately exciting each individual element with certain amplitudes and phases.

Over the last decade, wireless technology has grown at a formidable rate, thereby creating new and improved services at lower costs. This has resulted in an increase in airtime usage and in the number of subscribers. Recently, smart antenna systems have been widely considered to provide interference reduction and improve the performance of wireless mobile communication. The term "smart antenna" reflects the antenna's ability to adapt to the communication

Romanian Ministry of Education, CNCS - UEFISCDI channel environment in which it operates. Smart antenna arrays with adaptive beamforming capability are very effective in the suppression of interference and multipath signals. One practical solution to this problem is to use spatial processing. Spatial processing is the central idea of adaptive antennas or smart-antenna systems [2].

In this paper we are focused on changing of the phase of currents injected in antenna elements in order to conclude relating to position control of the radiation pattern characteristic and directivity.

\section{ANTENNAARRAY RADIATION PATTERN}

An antenna radiation pattern or antenna pattern is defined as "mathematical function or a graphical representation of the radiation properties of the antenna as a function of space coordinates. In most cases, the radiation pattern is determined in the far-field region and is represented as a function of the directional coordinates." [3] The radiation property of most concern is the two- or threedimensional spatial distribution of radiated energy as a function of the observer's position along a path or surface of constant radius.

Antennas with a given radiation pattern may be arranged in a structure (line, plane, three - dimensional) to yield a different radiation pattern. Given an antenna array of identical elements, the radiation pattern of the antenna array may be found according to the pattern multiplication theorem [4]:

\section{Array pattern $=$ Array element pattern $x$ Array factor}

where Array element pattern is the pattern of the individual array element and Array factor is a function dependent only on the geometry of the array and the excitation (amplitude, phase) of the elements.

\section{A. Linear Array}

The array factor (AF) is independent of the antenna type assuming all of the elements are identical. Thus, isotropic radiators may be utilized in the derivation of the array factor to simplify the algebra. The field of an isotropic radiator 
located at the origin may be written as (assuming $\theta$ polarization):

$$
E_{\theta}=I_{0} \frac{e^{-j k r}}{4 \pi r}
$$

where:

$I_{0}=$ the complex excitation of the isotropic radiator,

$k=$ the free space wave number,

$r=$ distance of the observation point from the origin.

In our approach, we assume that the elements of the array are uniformly - spaced with a separation distance $d$ (see Fig. $1)$.

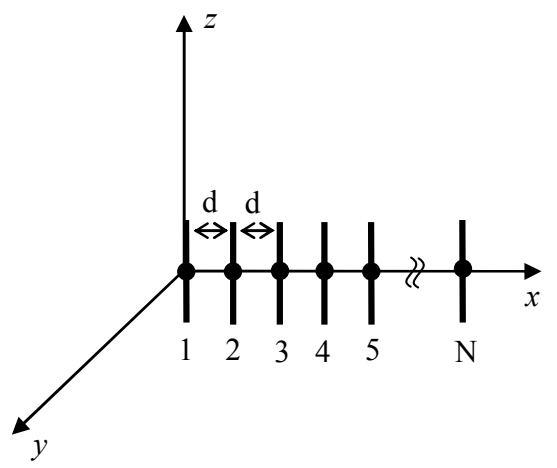

Fig. 1 The linear array antennas

The current magnitudes of the array elements are supposed to be equal and the current on the array element located at the origin is used as the phase reference (zero phase).

$$
I_{1}=I_{0} \quad I_{2}=I_{0} e^{j \phi_{2}} \quad \cdots \quad I_{N}=I_{0} e^{j \phi_{N}}
$$

The far electromagnetic fields of the individual array elements are:

$$
\begin{aligned}
& E_{\theta_{1}} \approx I_{0} \frac{e^{-j k r}}{4 \pi r}=E_{0} \\
& E_{\theta_{2}} \approx I_{0} e^{j \phi_{2}} \frac{e^{-j k(r-d \cos \theta)}}{4 \pi r}=E_{0} e^{j\left(\phi_{2}+k d \cos \theta\right)} \\
& \vdots \\
& E_{\theta_{N}} \approx I_{0} e^{j \phi_{N}} \frac{e^{-j k[r-(N-1) d \cos \theta]}}{4 \pi r}=E_{0} e^{j\left[\phi_{N}+(N-1) k d \cos \theta\right]}
\end{aligned}
$$

The overall array far field is found using superposition and could be express as:

$$
E_{\theta}=E_{\theta_{1}}+E_{\theta_{2}}+E_{\theta_{3}}+\ldots+E_{\theta_{N}}=E_{0} * A F
$$

The array factor for a uniformly - spaced $N$ - element linear array from (4) is:

$$
A F=\left[1+e^{j\left(\phi_{2}+k d \cos \theta\right)}+\ldots+e^{j\left[\phi_{N}+(N-1) k d \cos \theta\right]}\right]
$$

A uniform array is defined by uniformly - spaced identical elements of equal magnitude with a linearly progressive phase from element to element:

$$
\phi_{1}=0 \quad \phi_{2}=\alpha \quad \phi_{3}=2 \alpha \cdots \phi_{N}=(N-1) \alpha
$$

Inserting this linear phase progression into the formula for the general $N$ - element array, we obtain:

$$
A F=\left[1+e^{j(\alpha+k d \cos \theta)}+\ldots+e^{j(N-1)[\alpha+k d \cos \theta]}\right]=\sum_{n=1}^{N} e^{j(n-1) \psi}
$$

The function $\psi(\psi=\alpha+k d \cos \theta)$ is defined as the array phase function and is a function of the element spacing, phase shift, frequency and elevation angle. If the array factor from (5) is multiplied by $e^{j \psi}$, the result is:

$$
A F^{*} e^{j \psi}=\left[e^{j \psi}+e^{2 j \psi}+e^{3 j \psi}+\ldots+e^{j N \psi}\right]
$$

Substracting the array factor from the equation above, we obtain:

$$
A F=e^{j(N-1) \frac{\psi}{2}} \frac{\sin \left(\frac{N \psi}{2}\right)}{\sin \left(\frac{\psi}{2}\right)}
$$

The complex exponential term in (9) represents the phase shift of the array phase center relative to the origin. If the position of the array is shifted so that the center of the array is located at the origin, this phase term goes away. The array factor after phase shift and normalization becomes:

$$
A F=\frac{1}{N} \frac{\sin \left(\frac{N \psi}{2}\right)}{\sin \left(\frac{\psi}{2}\right)}
$$

\section{B. Planar Array}

Unlike linear arrays that can only scan the main beam in one polar plane ( $\theta$ - the elevation plane or $\phi$ - the azimuth plane), planar arrays scan the main beam along both $\theta$ and $\phi$. Planar arrays offer more gain and lower sidelobes than linear arrays, at the expense of using more elements. The design principles for planar arrays are similar to those presented earlier for the linear arrays. Since the elements are placed in two dimensions (see Fig. 2), the array factor of a planar array can be expressed as the multiplication of the array factors of two linear arrays [5]: one along the $\mathrm{x}$-axis and one along the y-axis:

$$
A F_{\text {planar }}=A F_{x} * A F_{y}=\frac{\sin \left(\frac{N_{1} \psi_{x}}{2}\right)}{N_{1} \sin \left(\frac{\psi_{x}}{2}\right)} \frac{\sin \left(\frac{N_{2} \psi_{y}}{2}\right)}{N_{2} \sin \left(\frac{\psi_{y}}{2}\right)}
$$

where:

$$
\begin{aligned}
& \psi_{x}=k d_{x} \sin \theta \cos \phi+\alpha_{x} \\
& \psi_{y}=k d_{y} \sin \theta \sin \phi+\alpha_{y}
\end{aligned}
$$




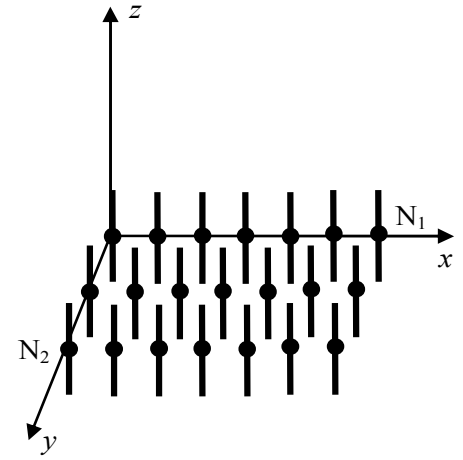

Fig. 2 The planar array antennas

\section{Three - dimensional Array (3 - D Array)}

Starting from the planar array, where it is considered that the system has a rectangular configuration of elements, the three - dimensional array antenna (Fig. 3) is achieved by introducing a number of planar arrays on the $z$ axis. In this case, the array factor is:

$$
\begin{aligned}
& A F_{3 D}=A F_{x} * A F_{y} * A F_{z}= \\
& =\frac{\sin \left(\frac{N_{1} \psi_{x}}{2}\right)}{N_{1} \sin \left(\frac{\psi_{x}}{2}\right)} \frac{\sin \left(\frac{N_{2} \psi_{y}}{2}\right)}{N_{2} \sin \left(\frac{\psi_{y}}{2}\right)} \frac{\sin \left(\frac{N_{3} \psi_{z}}{2}\right)}{N_{3} \sin \left(\frac{\psi_{z}}{2}\right)}
\end{aligned}
$$

where:

$$
\begin{aligned}
& \psi_{x}=k d_{x} \sin \theta \cos \phi+\alpha_{x} \\
& \psi_{y}=k d_{y} \sin \theta \sin \phi+\alpha_{y} \\
& \psi_{z}=k d_{z} \cos \theta+\alpha_{z}
\end{aligned}
$$

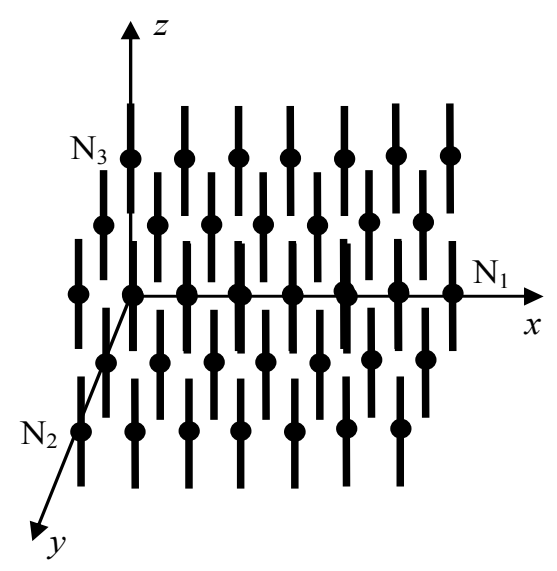

Fig. 3 Three - dimensional array antennas

In case of three - dimensional array antennas there are restrictions regarding the distance between elements on $z$ axis which could not be less than elements dimensions.

\section{SimUlation RESUltS}

Our simulations are made for linear, planar and three dimensional array antennas. Starting from a linear array antennas with logarithmic directivity pattern, composed between $2-9$ elements (dipole antenna) that are evenly spaced with the distance of $\lambda / 2$, we built a planar array and, respectively three - dimensional array antennas.

The simulations are made in Matlab and all results are displayed normalized. The radiation patterns obtained in different situations are generated in two planes, $x O y$ plane $(H$ plane) and $x O z$ plane ( $E$ plane). We use the opening angle of the main lobe to compare the results obtained in these situations [7].

Because for a linear array antenna the increases in a phase shift between currents injected in two consecutive elements leads to decreases in directivity, we are focused on planar and $3 D$ array antennas.

The radiation patterns obtained in $x O y$ plane ( $H$ plane) for planar array antennas with different number of elements and phases are represented in Fig. 4. In Fig. 4.c a radiation pattern for $45^{\circ}$ phase shift of currents injected in array elements is presented.

The radiation pattern obtained in $x O z$ plane ( $E$ plane) is presented in Fig. 5.

For planar array antenna with $7 \times 7$ elements, and three dimensional array antenna with $7 \times 7 \times 7$ elements, and $0^{\circ}$ phase shift, the radiation patterns are presented in Fig. 6.

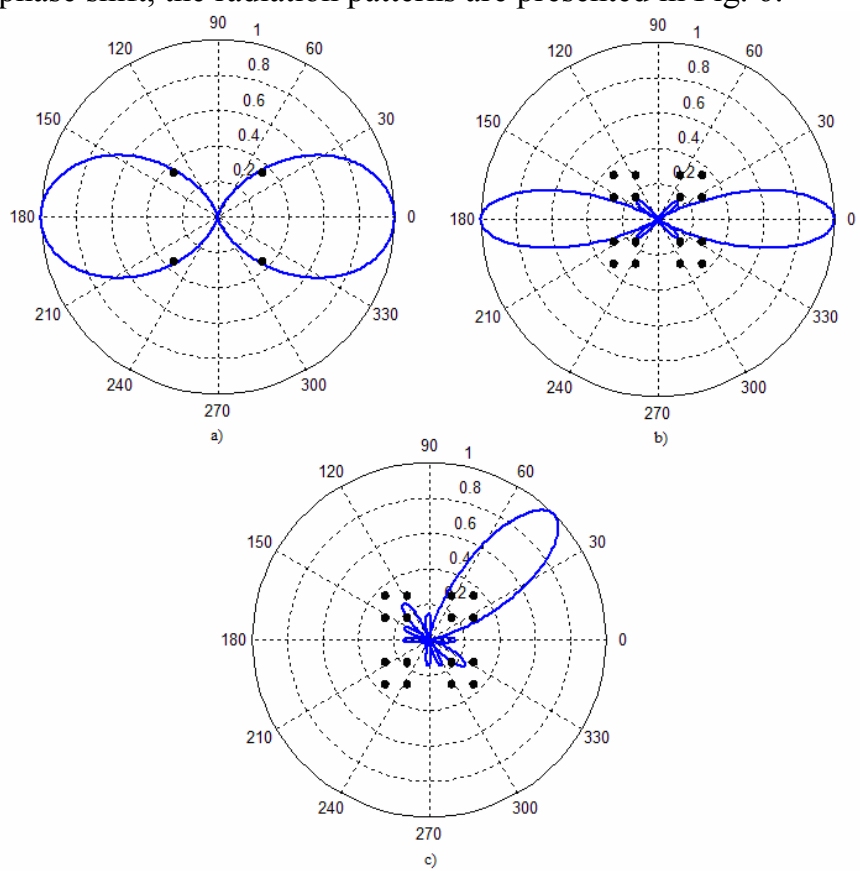

Fig. 4 Radiation patterns obtained in $H$ plane for planar array antennas: a) with $2 \times 2$ elements and $0^{\circ}$ phase shift; b) with $4 \times 4$ elements and $0^{\circ}$ phase shift; c) with $4 \times 4$ elements and $45^{\circ}$ phase shift 


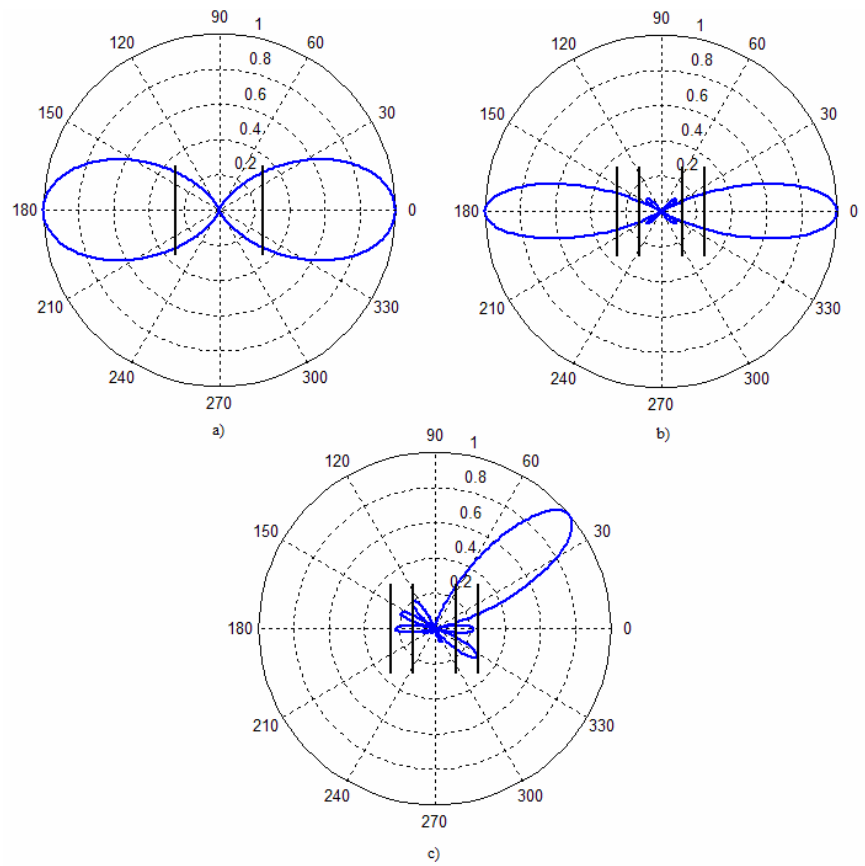

Fig. 5 Radiation patterns obtained in $E$ plane for planar array antennas: a) with $2 \times 2$ elements and $0^{\circ}$ phase shift; b) with $4 \times 4$ elements and $0^{\circ}$ phase shift; c) with $4 \times 4$ elements and $45^{\circ}$ phase shift
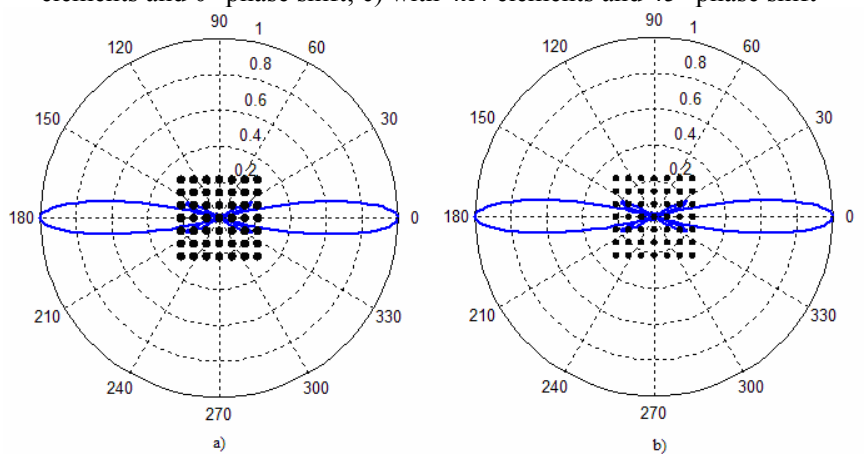

Fig. 6 Radiation patterns obtained in $H$ plane for: a) planar array antennas with $7 \times 7$ elements and; b) 3D array antennas with $7 \times 7 \times 7$ elements

As a comparison we used the opening angle of the main lobe. In case of identical currents injected in array elements the results are presented in Table I.
During the experiments we conclude that increasing the number of elements over 7 does not lead to significant increase of directivity, so we suggest that the maximum number of elements for an array should not be greater than 7 . Using planar array antennas with $7 \times 7$ elements and introducing a phase shift of $30^{\circ}$ and $165^{\circ}$ between the currents injected in two consecutive elements, we obtain the radiation patterns represented in Fig. 7. For three dimensional array antennas with $7 \times 7 \times 7$ elements and with a phase shift equal to $105^{\circ}$ and $165^{\circ}$ respectively, the radiation patterns are represented in Fig. 8.

The 3D patterns for planar array and three - dimensional array are presented in Fig. 9. Because we used a distance between elements equal with $\lambda / 2$ for phase shift equal with $180^{\circ}$ the obtained pattern is same as in case of no phase shift.

The values obtained for the opening angle of main lobe in case of array antennas formed by 7 elements with different phase shift between consecutive elements are presented in Table II.

We could observe that the opening angle for different structure array is same if the phase shift is complement to $180^{\circ}$, but for angles lower than $30^{\circ}$ or higher than complement to $180^{\circ}$ or for angles between $70^{\circ}$ and $110^{\circ}$ the pattern characteristic has two lobes, one in direction determined by phase shift and another to the direction complement to $180^{\circ}$ attenuated with $20 \%$.
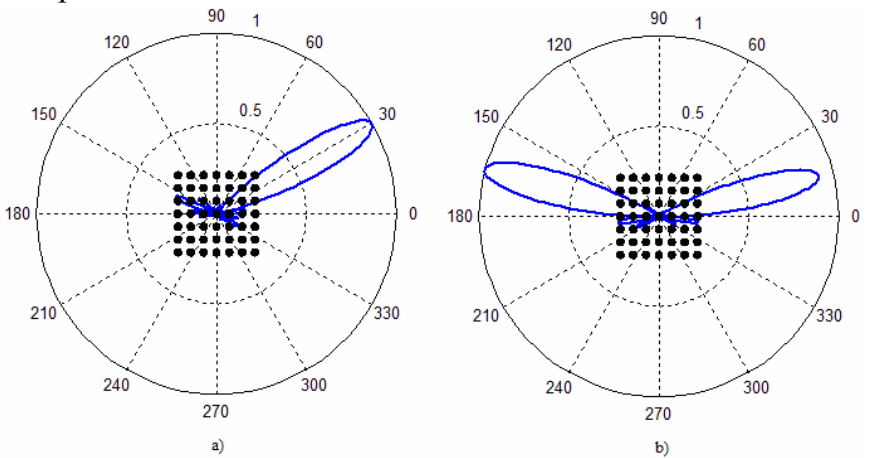

Fig. 7 Radiation pattern characteristic obtained in $H$ plane for planar array antennas with: a) $30^{\circ}$ phase shift, b) $165^{\circ}$ phase shift

TABLE I.

OPENING ANGLE for ARRAy ANTENNAS FOR Number of ELEMENTS

\begin{tabular}{|c|c|c|c|c|c|c|}
\hline \multirow{2}{*}{$\begin{array}{c}\text { Number of } \\
\text { elements }\end{array}$} & \multicolumn{2}{|c|}{ Linear } & \multicolumn{2}{c|}{ Planar } & \multicolumn{2}{c|}{ Three - dimensional } \\
\cline { 2 - 7 } & $\boldsymbol{H}$ & $\boldsymbol{E}$ & $\boldsymbol{H}$ & $\boldsymbol{E}$ & $\boldsymbol{H}$ & $27^{\circ}$ \\
\hline 2 & $59,6^{\circ}$ & $97,2^{\circ}$ & $58,4^{\circ}$ & $47^{\circ}$ & $58,4^{\circ}$ & $32,1^{\circ}$ \\
\hline 3 & $35,5^{\circ}$ & $57,3^{\circ}$ & $35,5^{\circ}$ & $32,1^{\circ}$ & $35,5^{\circ}$ & $12,6^{\circ}$ \\
\hline 4 & $25,3^{\circ}$ & $23,5^{\circ}$ & $26,3^{\circ}$ & $24^{\circ}$ & $26,3^{\circ}$ & $19,5^{\circ}$ \\
\hline 5 & $19,6^{\circ}$ & $47^{\circ}$ & $20,6^{\circ}$ & $19,5^{\circ}$ & $20,6^{\circ}$ & $9,1^{\circ}$ \\
\hline 6 & $16,1^{\circ}$ & $18,3^{\circ}$ & $17,1^{\circ}$ & $16^{\circ}$ & $17,1^{\circ}$ & $13,7^{\circ}$ \\
\hline 7 & $13,7^{\circ}$ & $40,1^{\circ}$ & $13,7^{\circ}$ & $13,7^{\circ}$ & $13,7^{\circ}$ & $6,8^{\circ}$ \\
\hline 8 & $11,4^{\circ}$ & $14,9^{\circ}$ & $11,4^{\circ}$ & $11,4^{\circ}$ & $11,4^{\circ}$ & $10,3^{\circ}$ \\
\hline 9
\end{tabular}



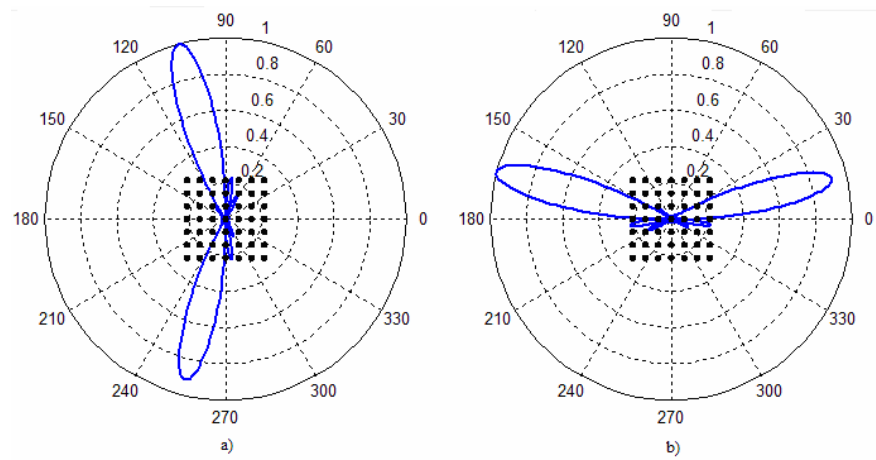

Fig. 8 Radiation pattern characteristic obtained in $H$ plane for three dimensional array antennas with: a) $105^{\circ}$ phase shift, b) $165^{\circ}$ phase shift
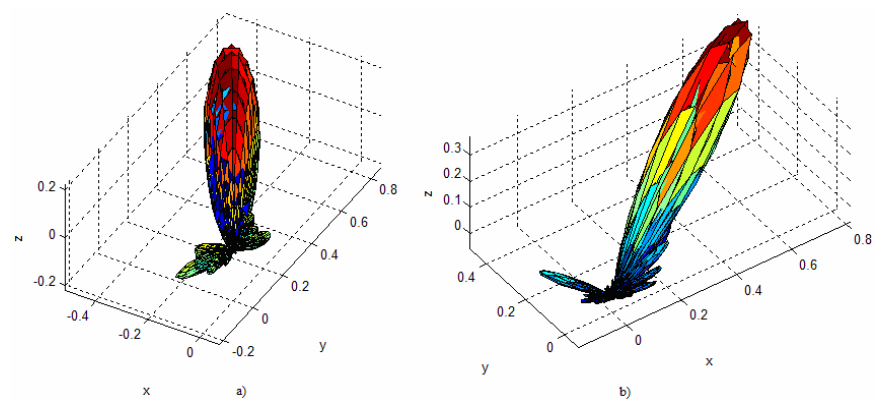

Fig. $93 \mathrm{D}$ radiation pattern characteristic for: a) planar array antennas with $120^{\circ}$ phase shift, b) three - dimensional array antennas with $30^{\circ}$ phase shift

\section{WSN APPLICATIONS}

One of the most interesting applications for directional antenna is Wireless Sensor Networks (WSN). WSN represent a spatially distributed of large number of autonomous sensors which collect data from environment and is able to send it to a main certain locations. WSN has many applications from different field. The main areas of applications of WSN are: military (monitoring forces, equipment and ammunitions; battlefield surveillance; targeting, etc.), environmental (forest fire detection; biocomplexity mapping of environment; flood detection; air and water pollution), health (telemonitoring of human physiological data, tracking and monitoring doctors and patients, drug administration), home and office (automation; smart environment), automotive (reduces wiring effects; measurements in chambers and rotating parts, remote technical inspections, conditions monitoring e.g. at a bearing) [8].

The architecture of the WSNs can take many forms starting from a star network to an advanced multi-hop wireless network. One possible solution for WSN communications architecture is presented in Fig. 10.

The components of the networks are called nodes; each of them is connected to one or, sometimes, several others. The main structure for sensor nodes is presented in Fig. 11.

In many cases in order to connect to send data it is necessary to obtain a maximum signal to noise ratio in transmission [9].

Because the transmission system has a consumption that depends on the signal to noise ratio, it is necessary to improve this parameter.

In a transmission system the signal to noise ratio could be improved without increasing energy if we are able to increase the gain of the antenna or if we are able to orient the antennas in a way in what the pattern characteristics are aligned. Using an array antenna we can control the orientation of the pattern characteristic without any energy consumption. This leads to the increased signal to noise ratio and to the reduced energy consumption because it is not necessary a mechanical orientation of the elements of the node in order to obtain maximum signal to noise ratio.

TABLE II.

Opening Angle for ArRay Antennas for Phase ShifT

\begin{tabular}{|c|c|c|c|c|c|c|}
\hline \multirow{2}{*}{ Phase shift } & \multicolumn{2}{|c|}{ Linear } & \multicolumn{2}{c|}{ Planar } & \multicolumn{2}{c|}{ Three - dimensional } \\
\cline { 2 - 7 } & $\boldsymbol{H}$ & $\boldsymbol{E}$ & $\boldsymbol{H}$ & $\boldsymbol{E}$ & $\boldsymbol{H}$ & $\boldsymbol{E}$ \\
\hline $0^{0}$ & $13,7^{\circ}$ & $40,1^{\circ}$ & $13,7^{\circ}$ & $13,7^{\circ}$ & $13,7^{\circ}$ & $13,7^{\circ}$ \\
\hline $15^{\circ}$ & $14,9^{\circ}$ & $32,1^{\circ}$ & $13,7^{\circ}$ & $13,7^{\circ}$ & $13,7^{\circ}$ & $12,6^{\circ}$ \\
\hline $30^{\circ}$ & $13,7^{\circ}$ & $11,4^{\circ}$ & $13,7^{\circ}$ & $13,7^{\circ}$ & $13,7^{\circ}$ & $10,3^{\circ}$ \\
\hline $45^{\circ}$ & $14,9^{\circ}$ & $12,6^{\circ}$ & $14,9^{\circ}$ & $13,7^{\circ}$ & $14,9^{\circ}$ & $10,3^{\circ}$ \\
\hline $60^{\circ}$ & $14,9^{\circ}$ & $13,7^{\circ}$ & $14,9^{\circ}$ & $13,7^{\circ}$ & $14,9^{\circ}$ & $10,3^{\circ}$ \\
\hline $75^{0}$ & $16^{\circ}$ & $13,7^{\circ}$ & $14,9^{\circ}$ & $12,6^{\circ}$ & $14,9^{\circ}$ & $10,3^{\circ}$ \\
\hline $90^{\circ}$ & $17,2^{\circ}$ & $13,7^{\circ}$ & $13,7^{\circ}$ & $10,5^{\circ}$ & $13,7^{\circ}$ & $12,6^{\circ}$ \\
\hline $105^{0}$ & $16^{\circ}$ & $13,7^{\circ}$ & $14,9^{\circ}$ & $12,6^{\circ}$ & $14,9^{\circ}$ & $10,3^{\circ}$ \\
\hline $120^{\circ}$ & $14,9^{\circ}$ & $13,7^{\circ}$ & $14,9^{\circ}$ & $13,7^{\circ}$ & $14,9^{\circ}$ & $10,3^{\circ}$ \\
\hline $135^{0}$ & $14,9^{\circ}$ & $12,6^{\circ}$ & $14,9^{\circ}$ & $13,7^{\circ}$ & $14,9^{\circ}$ & $10,3^{\circ}$ \\
\hline $150^{\circ}$ & $13,7^{\circ}$ & $11,4^{\circ}$ & $13,7^{\circ}$ & $13,7^{\circ}$ & $13,7^{\circ}$ & $10,3^{\circ}$ \\
\hline $165^{\circ}$ & $14,9^{\circ}$ & $32,1^{\circ}$ & $13,7^{\circ}$ & $13,7^{\circ}$ & $13,7^{\circ}$ & $12,6^{\circ}$ \\
\hline $180^{\circ}$ & $13,7^{\circ}$ & $40,1^{\circ}$ & $13,7^{\circ}$ & $13,7^{\circ}$ & $13,7^{\circ}$ & $13,7^{\circ}$ \\
\hline
\end{tabular}




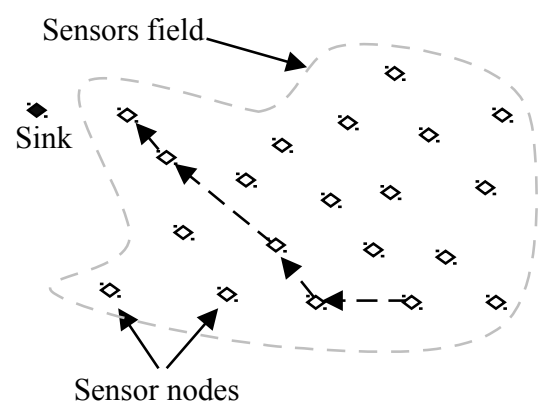

Fig. $10 \mathrm{WSN}$ architecture

\begin{tabular}{|c|}
\hline Sensor unit \\
\hline $\begin{array}{c}\text { Processor } \\
\text { unit }\end{array}$ \\
\hline $\begin{array}{c}\text { Transceiver } \\
\text { unit }\end{array}$ \\
\hline Power unit \\
\hline
\end{tabular}

Fig. 11 Node architecture

\section{CONCLUSION}

A brief overview of antenna array aspects has been given. We observe that in cases of planar array and three - dimensional array, the systems become more directly. Also, if a phase shift between current injected in the antenna elements is introduced the linear array become impracticable in terms of directivity pattern.

During the experiments we conclude that increasing the number of elements over 7 does not lead to significant increase of directivity, so we suggest that the maximum number of elements for an array should not be greater than 7 .

In order to obtain an oriented pattern characteristic, only planar and 3D array could be used because a linear array has many secondary lobes, and in this case the gain decreases. Also in order to obtain a good directivity the phase shift should be between 30 and 150 degree.
Array antennas are increasingly used nowadays because using them can improve radio transmissions by:

- increasing signal coverage, by increasing capacity for data transfer,

- reducing interference due to multipath signal propagation, simultaneous multiple signals radiated to different users,

- better focus of the beam radiated to the direction of interest.

For a better orientation of the beam radiated to a user, the array antenna must be able to detect the direction from which the signal arrives from the user. Future work is oriented to investigate what is happened in case of changes the regularity of the array structure and also a new structure for planar array.

\section{ACKNOWLEDGMENT}

The work of Horia Baltă was supported by a grant of the Romanian Ministry of Education, CNCS - UEFISCDI, project number PN-II-RU-PD-2012-3-0122. Horia Baltă is also with Valahia University of Târgovişte, Romania.

\section{REFERENCES}

[1] C.A. Balanis, Antenna Theory: Analysis and Design. $2^{\text {nd }}$ ed., John Wiley \& Sons, New York, 1997, pp. 27-48.

[2] W.L. Stutzmann, G.A. Thiele, Antenna Theory and Design. John Wiley \& Sons, New York, 1998, pp. 31-35.

[3] R.J. Mailloux, Phased Array Antenna Handbook. $2^{\text {nd }}$ ed., Artech House, New York, 2005, pp. 48-100.

[4] F.T. Ulaby, Applied Electromagnetics. Prentice Hall, Inc., New Jersey, 2007, pp. $100-125$.

[5] C.A. Balanis, Modern Antenna Handbook, John Wiley \& Sons, New York, 2008, pp. 60-95.

[6] K. K. Verma, K. R. Soni, "Theoretical study of $2 \times 2$ element planar array of equilateral triangular patch microstrip antenna in plasma medium," PRAMANA - Journal of Physics, vol. 64, pp. 147-152, Jan. 2005.

[7] A. Ignea, E. Marza, A. De Sabata, Antene şi Propagare (in romanian language). Editura de Vest, Timişoara, 2002, pp. 289-310.

[8] W. Dargie, C. Poellabauer, Fundamentals of wireless sensor networks: theory and practice, John Wiley \& Sons Ltd, New York, 2010, pp. $150-245$

[9] P. Dorfinger, G. Panholzer, F. von Tüllenburg, M. Cristaldi, G. Tusa, and F. Böhm, Self-aligning wireless communication for first responder organizations in interoperable emergency scenarios, ICN 2015, Proceedings of The Fourteenth International Conference on Networks, April 19 - 24, 2015, Barcelona, Spain, pp.230-236, 